\title{
Existence Results for Nonlinear Quasi-hemivariational Inequality Systems
}

https://doi.org/10.32792/utq/uti/vol11/4/7

\author{
Ayed E. Hashoosh , Mohsen Alimohammady \\ and Ghufran A. Almusawi \\ Department of Mathematics, University of Mazandaran, \\ Iran; Email: amohsen@umz.ac.ir \\ * Department of Mathematics, University of Thi-Qar, \\ Iraq; Email ghufranalmusawi21@gmail.com \\ ayed197991@yahoo.com
}

\section{ABSTRACT}

This paper aims at establishing the existence solutions of a non-standard quasi-hemivariational inequality, whose solutions are discussed in a subset $K_{n}$ of a reflexive Banach space $X_{n}$ for every $n=\overline{1,1}$. In addition, we introduce two applications. The first one shows the relationship between our inequality problems and equilibrium problems. The second one applies the extend some results concerning nonlinear quasi-hemivariational inequalities.

$$
\begin{aligned}
& \text { (الملخص }
\end{aligned}
$$

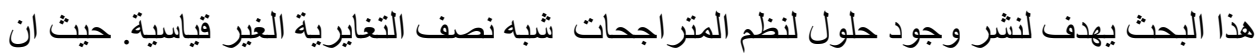

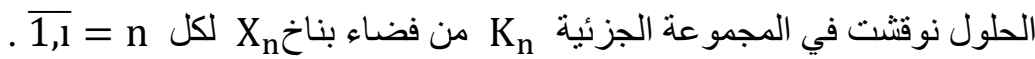

$$
\begin{aligned}
& \text { بالإضافة الى ذللك قدمنا تطبيقين. الاول بين مشاكل متر اجتنا ومشاكل التوازن ـ اما الثناني يطبق }
\end{aligned}
$$

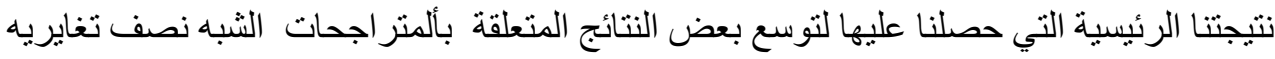

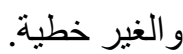

Keywords

Quasi-hemivariational inequality; Clarkes generalized gradient; locally Lipschitz functional; equilibrium problems.

Mathematics subject classification 2010: 47H04; 47H05; 47J20; 49J53; $26 \mathrm{D} 12$. 
University of Thi-Qar Journal Vol.11 No.4 DEC 2016

Web Site: https://jutq.utq.edu.iq/index.php/main Email: journal@jutq.utq.edu.iq 


\section{University of Thi-Qar Journal Vol.11 No.4 DEC 2016}

Web Site: https://jutq.utq.edu.iq/index.php/main Email: journal@jutq.utq.edu.iq

\section{Introduction}

The theory of hemivariational inequalities was introduced P.D.

Panayiotopoulos at the beginning of the 1980s (see [18] and [19]).

Within a very short period of time, this theory witnessed a remarkable development in both pure and applied mathematics. It has been proved very efficient to describe a variety of mechanical problems and engineering sciences, economics, differential inclusion and optimal control (see $[5-8,12,15,16,18,21,22]$ and [25]). In these papers, based on Clarkes

generalized directional derivative and Clarke's generalized gradient for locally Lipchitz functions, the researchers study the existence and uniqueness of solutions by using such as fixed point Theorems, KKM Theorems, critical point Theory, surjectivity Theorems for pseudomonotone and coercive operators (see $[1,3,4,11,13,14,20$, $23,24]$ and [26]).

Generally, in the last few years, there were many authors were who interested in the study of various kinds of hemivariational inequalities and systems of hemivariational inequalities, these inequalities are a generalization of the variational inequalities, and related problems such as equilibrium problems. In order to, it is very useful to understand several problems of mechanics and engineering for non- convex, nonsmooth energy functionals.

Quasi- hemivariational inequalities arise from hemivariational inequalities if in addition some constraints have to be taken into account. The solution of these inequality gives the position of the state equilibrium of the structure.

The main purpose of this work is to contribute in establishing the existence solutions for systems of generalized quasi- hemivariational inequalities. In order to achieve the aim, the study is divided into the following sections.

In section 2, we refer to some definitions and results that assist us in the study. In section 3 we formulate the system of generalized quasihemivariational inequality and prove the main results. In the last section of the paper, two applications are given. The first one present the relationship between our inequality problems and equilibrium problems. The second one apply of our inequality to a system of 


\section{University of Thi-Qar Journal Vol.11 No.4 DEC 2016}

Web Site: https://jutq.utq.edu.iq/index.php/main Email: journal@jutq.utq.edu.iq

generalized quasi- hemivariational inequalities involving integrals of Clarke's generalized directional derivatives.

\section{Preliminaries with basic assumptions}

Throughout this paper, we assume that $E_{n}$ are Banach space and that $E_{n}{ }^{*}$ are the topological dual space of the Banach space $E_{n}$, white $\langle\cdot, \cdot\rangle_{n}$ and $\|\cdot\|_{n}$ denote the duality pairing between $E_{n}$ and $E_{n}{ }^{*}$, respectively for every $n=\overline{1, l}$.

In what follows, we are going to recall some definitions and notions from nonsmooth analysis which will be used in this paper.

Assume that $\mathrm{K}$ is a nonempty, closed and convex subset of a Banach space $\mathrm{E}_{\mathrm{n}}$ and assume that $\mathrm{F}: \mathrm{K} \times \mathrm{K} \rightarrow \mathrm{R}$ us a given bifunction satisfying the property $F(u, u)=0$, for every $u \in K$. An equilibrium problem (for short, (EP)). In the sense of Blum, Mu and Zoettli (see [17]) is a problem of the form:

Find $x \in K$ such that $F(x, y) \geq 0, \forall y \in K$.

Definition 2.1: [8] A functional J: $\mathrm{E} \rightarrow \mathrm{R}$ is said to be locally Lipchitz if every point $\mathrm{u} \in \mathrm{E}$ possesses a neighborhooh $\mathrm{W}$ such that

$$
|J(a)-J(b)| \leq M_{u}\|a-b\|_{E} \forall a, b \in W .
$$

For a constant $\mathrm{M}_{\mathrm{u}} \geq 0$ which depends on $\mathrm{W}$.

Definition 2.2: [8] Assume that J: $\mathrm{E} \rightarrow \mathrm{R}$ is a locally Lipchitz. The generalized derivative of $\mathrm{J}$ at the point $\mathrm{u} \in \mathrm{E}$ in the direction $\mathrm{z} \in \mathrm{E}$ is denoted by $\mathrm{J}^{0}(\mathrm{u}, \mathrm{z})$, i.e.,

$$
\mathrm{J}^{0}(\mathrm{u}, \mathrm{z})=\operatorname{limsupa}_{\lambda \rightarrow 0} \frac{\mathrm{J}(\mathrm{a}+\lambda \mathrm{z})-\mathrm{J}(\mathrm{a})}{\lambda} .
$$

Similarly, one can define the partial generalized derivative and partial generalized gradient of locally Lipchitz functional in the $\mathrm{r}^{\text {th }}$ variable.

Definition 2.3: [8] Assume that $\emptyset: E_{1} \times \ldots \times E_{r} \times \ldots E_{n} \rightarrow R$ be a locally lipschitz function in the $\mathrm{r}^{\text {th }}$ variable. The partial generalized derivative of 


\section{University of Thi-Qar Journal Vol.11 No.4 DEC 2016}

Web Site: https://jutq.utq.edu.iq/index.php/main Email: journal@jutq.utq.edu.iq

$\emptyset\left(\mathrm{u}_{1}, \ldots, \mathrm{u}_{\mathrm{r}}, \ldots, \mathrm{u}_{\mathrm{i}}\right)$ at the point $\mathrm{u}_{\mathrm{r}} \in \mathrm{E}_{\mathrm{r}}$ in the direction $\mathrm{z}_{\mathrm{r}} \in \mathrm{E}_{\mathrm{r}}$, denoted by $\emptyset^{0}\left(u_{1}, \ldots, u_{r}, \ldots, u_{i} ; z_{r}\right)$, is

$$
\emptyset^{0}{ }_{r}\left(\mathrm{u}_{1}, \ldots, \mathrm{u}_{\mathrm{r}}, \ldots, \mathrm{u}_{\mathrm{i}} ; \mathrm{z}_{\mathrm{r}}\right)=\limsup _{\substack{\mathrm{r} \\ \lambda\lrcorner 0}} \frac{\emptyset\left(\mathrm{u}_{\mathrm{r}}, \ldots, \mathrm{a}_{\mathrm{r}}+\lambda \mathrm{z}_{\mathrm{r}}, \ldots, \mathrm{u}_{\mathrm{i}}\right)-\emptyset\left(\mathrm{u}_{1}, \ldots, \mathrm{a}_{\mathrm{r}}, \ldots, \mathrm{u}_{\mathrm{i}}\right)}{\lambda} .
$$

While the partial generalized gradient of the mapping

$\mathrm{u}_{\mathrm{r}} \mapsto \mathrm{f}\left(\mathrm{u}_{1}, \ldots, \mathrm{u}_{\mathrm{r}}, \ldots, \mathrm{u}_{\mathrm{i}}\right)$ denoted by $\partial_{\mathrm{r}} \emptyset(\mathrm{u})\left(\mathrm{u}_{1}, \ldots, \mathrm{u}_{\mathrm{r}}, \ldots, \mathrm{u}_{\mathrm{i}}\right), \forall \mathrm{z}_{\mathrm{r}} \in \mathrm{E}_{\mathrm{r}}$ that is

$\partial_{\mathrm{r}} \emptyset(\mathrm{u})\left(\mathrm{u}_{1}, \ldots, \mathrm{u}_{\mathrm{r}}, \ldots, \mathrm{u}_{\mathrm{i}}\right)=\left\{\mathrm{s}_{\mathrm{r}} \in \mathrm{E}_{\mathrm{r}}^{*}: \emptyset^{0}{ }_{, \mathrm{r}}\left(\mathrm{u}_{1}, \ldots, \mathrm{u}_{\mathrm{r}}, \ldots, \mathrm{u}_{\mathrm{i}} ; \mathrm{z}_{\mathrm{r}}\right) \geq\left\langle\varsigma_{\mathrm{r}}, \mathrm{z}_{\mathrm{r}}\right\rangle \mathrm{E}_{\mathrm{r}}\right\}$.

Definition2.4: [8] Assume that $\mathrm{E}$ is a Banach space and $\mathrm{J}: \mathrm{E} \rightarrow \mathrm{R}$ is locally lipschitz functional. We say that $\mathrm{J}$ is a regular (in the sense of Clarkes) at $\mathrm{u} \in \mathrm{E}$ if for each $z \in E$ the one sided directional derivative $J^{\prime}(u, z)$ exists and $\mathrm{J}^{0}(\mathrm{u}, \mathrm{z})=\mathrm{J}^{\prime}(\mathrm{u}, \mathrm{z})$. We say that $\mathrm{J}$ is regular at every point $\mathrm{u} \in \mathrm{E}$.

Proposition 2.5: [8] Let $\mathrm{J}: \mathrm{E} \rightarrow \mathrm{R}$ be a function on a Banach space $\mathrm{E}$, which is locally Lipchitz of rank $M_{u}$ near the point $z \in E$, then

i) The $\mathrm{z} \mapsto \mathrm{J}^{0}(\mathrm{u}, \mathrm{z})$ is subadditive, finite, positively homogeneous and satisfies

ii) $\quad \mathrm{J}^{0}(\mathrm{u}, \mathrm{z}) \leq \mathrm{M}_{\mathrm{u}}\|\mathrm{z}\|$;

iii) $\quad J^{0}(u, z)$ is upper semicontinuous as a function of $(u, z)$ one can found a proof it in [19].

Definition 2.6: The generalized gradient of $\mathrm{J}$ at $\mathrm{u} \in \mathrm{E}$, which is a subset of a dual space $\mathrm{E}^{*}$, is define by

$$
\partial \mathrm{J}(\mathrm{u})=\left\{\xi \in \mathrm{E}^{*}:\langle\xi, \mathrm{z}\rangle \leq \mathrm{J}^{0}(\mathrm{u} ; \mathrm{z}), \forall \mathrm{z} \in \mathrm{E}\right\} .
$$

Lemma 2.7. [8] Assume that $\emptyset: \mathrm{E}_{1} \times \ldots \times \mathrm{E}_{\mathrm{i}} \rightarrow \mathrm{R}$ is a regular, locally Lipchitz functional. Then the following are satisfied:

(1) $\partial \emptyset\left(\mathrm{u}_{1}, \ldots, \mathrm{u}_{\mathrm{r}}, \ldots, \mathrm{u}_{\mathrm{i}}\right) \subset$ $\partial_{1} \varnothing\left(u_{1} \ldots, u_{r}, \ldots, u_{i}\right) \ldots \times \partial_{i} \varnothing(u)\left(u_{1}, \ldots, u_{r}, \ldots, u_{i}\right) ;$

(2) $\emptyset\left(\mathrm{u}_{1}, \ldots, \mathrm{u}_{\mathrm{r}}, \ldots \mathrm{u}_{\mathrm{i}}, \mathrm{z}_{1} \ldots, \mathrm{z}_{\mathrm{r}}, \ldots, \mathrm{z}_{\mathrm{i}}\right) \leq \sum_{\mathrm{i}=1}^{\mathrm{n}} \emptyset^{0}{ }_{\mathrm{r}}\left(\mathrm{u}_{1}, \ldots, \mathrm{u}_{\mathrm{r}}, \ldots, \mathrm{u}_{\mathrm{i}}\right)$;

(3) $\emptyset\left(u_{1}, \ldots, u_{r}, \ldots, u_{i}, 0, \ldots, z_{r}, \ldots, 0\right) \leq \emptyset^{0}{ }_{r}\left(u_{1}, \ldots, u_{r}, \ldots, u_{i} ; z_{r}\right)$. 


\section{University of Thi-Qar Journal Vol.11 No.4 DEC 2016}

Web Site: https://jutq.utq.edu.iq/index.php/main Email: journal@jutq.utq.edu.iq

Definition2.8. Assume that $\mathrm{T}: \mathrm{E} \rightarrow \mathrm{E}^{*}$ and $\eta: \mathrm{E} \times \mathrm{E} \rightarrow \mathrm{E}$ are two single valued. $T$ is said to be $\eta$ - monotone, if for each $x, y \in \operatorname{dom} T$ such that

$$
\langle\mathrm{Tx}-\mathrm{Ty}, \eta(\mathrm{x}, \mathrm{y})\rangle \geq 0 .
$$

At the end of this section, we recall Tarafdar fixed point theorem for set valued mapping [25] which we shall use to prove the main results of the study.

Theorem 2.9. Assume that $\mathrm{K} \subset \mathrm{E}$ is a nonempty and convex of Hausdorff topology vector space $\mathrm{E}$, and that $\alpha: \mathrm{K} \multimap \mathrm{K}$ be a set valued map such the following are satisfied

(i) For each $u \in K, \alpha(u)$ is a nonempty convex subset of $K$.

(ii) For each $\mathrm{z} \in \mathrm{K}, \alpha^{-1}(\mathrm{z})=\{\mathrm{u} \in \mathrm{K}: \mathrm{z} \in \alpha(\mathrm{u})\}$ contain an open set $\mathrm{O}_{\mathrm{z}}$ which may be empty.

(iii) $\mathrm{U}_{\mathrm{z} \in \mathrm{K}} \mathrm{O}_{\mathrm{z}}=\mathrm{K}$.

(iv) There exists a nonempty set $\mathrm{U}_{0}$ contained in a compact subset $\mathrm{U}_{1}$ of $\mathrm{K}$ such that $\mathrm{S}=\bigcap_{\mathrm{z} \in \mathrm{K}} \mathrm{O}_{\mathrm{z}}{ }^{\mathrm{c}}$ is either empty or compact, where $\mathrm{O}_{\mathrm{z}}{ }^{\mathrm{c}}$ is the complement of $\mathrm{O}_{\mathrm{z}} \in \mathrm{K}$.

Then there exists a point $u^{0} \in K$ such that $u^{0} \in \alpha\left(u^{0}\right)$.

\section{Formulation of the problem and main results:}

Assume that $K_{n}$ is a nonempty bounded, closed and convex subset of a real reflexive Banach space $X_{n}$ where $n$ a positive integer. Our aim is to study the following system of nonlinear quasi- hemivariational inequality: (NQHIS)

$$
\begin{aligned}
& \text { Find }\left(\mathrm{u}_{1}, \ldots, \mathrm{u}_{\mathrm{i}}\right) \in \mathrm{K}_{1} \times \ldots \times \mathrm{K}_{\mathrm{i}} \text {. }
\end{aligned}
$$

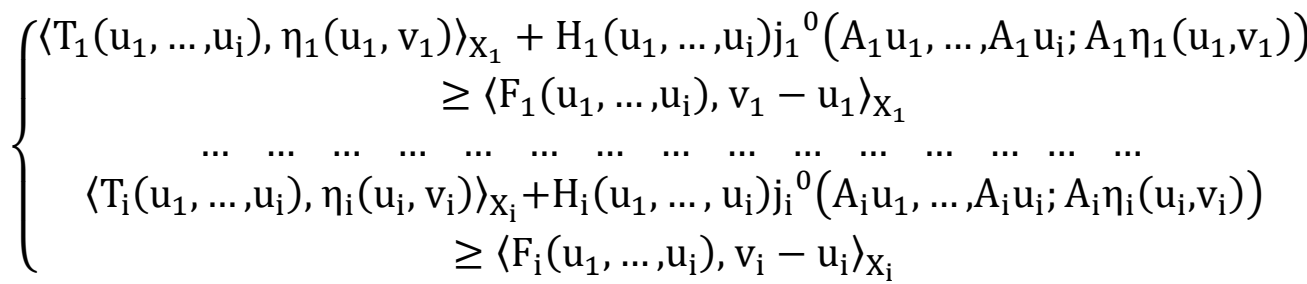

For all $\left(\mathrm{v}_{1}, \ldots, \mathrm{v}_{\mathrm{i}}\right) \in \mathrm{K}_{1} \times \ldots \times \mathrm{K}_{\mathrm{i}}$. Assume that $\mathrm{X}_{1}, \ldots, \mathrm{X}_{\mathrm{i}}$ are reflexive Banach space and that $\mathrm{E}_{1}, \ldots, \mathrm{E}_{\mathrm{i}}$ are Banach space. We assume that there exist compact 


\section{University of Thi-Qar Journal Vol.11 No.4 DEC 2016}

Web Site: https://jutq.utq.edu.iq/index.php/main Email: journal@jutq.utq.edu.iq

linear operators $A_{n}: X_{n} \rightarrow E_{n}$ for every $n=\overline{1,1}$. In addition, let us assume that $\eta_{\mathrm{n}}: \mathrm{X}_{\mathrm{n}} \times \mathrm{X}_{\mathrm{n}} \rightarrow \mathrm{X}_{\mathrm{n}}$ are single- valued functions, and J: $\mathrm{E}_{1} \times \ldots \times \mathrm{E}_{\mathrm{n}} \rightarrow \mathrm{R}$ is a regular locally Lipchitz functional, for every $n=\overline{1,1}$.

Notations: For every $n=\overline{1,1}$. Then

${ }^{\circ} \mathrm{X}=\mathrm{X}_{1} \times \ldots \times \mathrm{X}_{\mathrm{i}} ; \mathrm{E}=\mathrm{E}_{1} \times \ldots \times \mathrm{E}_{\mathrm{i}}$ and $\mathrm{E}=\mathrm{K}_{1} \times \ldots \times \mathrm{K}_{\mathrm{i}}$

${ }^{\circ} \mathrm{u}=\left(\mathrm{u}_{1}, \ldots, \mathrm{u}_{\mathrm{i}}\right)$ and $\mathrm{A}_{\mathrm{u}}=\left(\mathrm{A}_{1} \mathrm{u}_{1}, \ldots, \mathrm{A}_{\mathrm{i}} \mathrm{u}_{\mathrm{i}}\right)$

${ }^{\circ} \eta(u, v)=\left(\eta_{1}\left(u_{1}, v_{1}\right), \ldots, \eta_{i}\left(u_{i}, v_{i}\right)\right) ; A_{\eta}(u . v)$

$$
=\left(A_{1} \eta_{1}\left(u_{1}, v_{1}\right), \ldots, A_{i} \eta_{i}\left(u_{i}, v_{i}\right)\right)
$$

${ }^{\circ} \mathrm{T}_{\mathrm{n}}$ and $\mathrm{F}_{\mathrm{n}}: \mathrm{X}_{1} \times \ldots \times \mathrm{X}_{\mathrm{i}} \rightarrow \mathrm{X}_{\mathrm{n}}{ }^{*}$ are nonlinear operators such that:

$$
\begin{aligned}
& \left\langle F_{u}, v-u\right\rangle_{X}=\sum_{n=1}^{i}\left\langle F_{n}\left(u_{1}, \ldots, u_{i}\right), v_{n}-u_{n}\right\rangle_{X_{n}} \\
& \left\langle T_{u}, \eta(u, v)\right\rangle_{X}=\sum_{n=1}^{i}\left\langle T_{n}\left(u_{1}, \ldots, u_{i}\right), \eta_{n}\left(u_{n}, v_{n}\right)\right\rangle_{n}
\end{aligned}
$$

${ }^{\circ} \mathrm{H}_{\mathrm{n}}: \mathrm{X}_{1} \times \ldots \times \mathrm{X}_{\mathrm{i}} \rightarrow \mathrm{R} \cup\{+\infty\}$ is a non negative and continuous on $\mathrm{K}_{\mathrm{n}}$ such that $\mathrm{H}(\mathrm{u})=\sum_{\mathrm{n}=1}^{\mathrm{i}}\left(\mathrm{u}_{1}, \ldots, \mathrm{u}_{\mathrm{i}}\right)$.

Hypotheses: For every $n=\overline{1,1}$. Then the following assertions are fulfilled

$H_{F}:\left\langle F_{n}\left(u_{1}, \ldots, u_{i}\right), v_{n}-u_{n}\right\rangle_{X_{n}} \leq \liminf _{m}\left\langle F_{n}\left(u_{1}{ }^{m}, \ldots, u_{i}{ }^{m}\right), v_{n}-u_{n}{ }^{m}\right\rangle_{X_{n}}$,

Where $\left(\mathrm{u}_{1}{ }^{\mathrm{m}}, \ldots, \mathrm{u}_{\mathrm{i}} \mathrm{m}\right) \rightarrow\left(\mathrm{u}_{1}, \ldots, \mathrm{u}_{\mathrm{i}}\right)$ as $\mathrm{m} \rightarrow \infty$ and $\mathrm{v}_{\mathrm{n}} \in \mathrm{X}_{\mathrm{n}}$ is fixed.

$H_{\eta}$ : The mapping $\eta_{i}(\cdot, \cdot): X_{i} \times X_{i} \rightarrow X_{i}$ satisfied the following conditions

(1) $\eta_{\mathrm{n}}\left(\mathrm{u}_{\mathrm{n}}, \mathrm{u}_{\mathrm{n}}\right)=0 \quad \forall \mathrm{u}_{\mathrm{n}} \in \mathrm{X}_{\mathrm{n}}$;

(2) $\eta_{n}\left(u_{n}, 0\right)$ is a linear operator $\forall u_{n} \in X_{n}$;

(3) $\eta_{n}\left(u_{n}{ }^{m}, v_{n}\right) \rightarrow \eta\left(u_{n}, v_{n}\right)$, whenever $u_{n}{ }^{m} \rightarrow u_{n}$.

$\mathrm{H}_{\mathrm{T}}$ :

(1) $\limsup _{m}\left\langle\mathrm{~T}_{\mathrm{n}}\left(\mathrm{u}_{1}{ }^{\mathrm{m}}, \ldots, \mathrm{u}_{\mathrm{i}}^{\mathrm{m}}\right), \eta_{\mathrm{n}}\left(\mathrm{u}_{\mathrm{n}}^{\mathrm{m}}, \mathrm{v}_{\mathrm{n}}\right)\right\rangle_{\mathrm{x}_{\mathrm{n}}} \leq$ $\left\langle\mathrm{T}_{\mathrm{n}}\left(\mathrm{u}_{1}, \ldots, \mathrm{u}_{\mathrm{i}}\right), \eta_{\mathrm{n}}\left(\mathrm{u}_{\mathrm{n}}, \mathrm{v}_{\mathrm{n}}\right)\right\rangle_{\mathrm{X}_{\mathrm{n}}}$ where $\left(\mathrm{u}_{1} \mathrm{~m}, \ldots, \mathrm{u}_{\mathrm{i}}{ }^{\mathrm{m}}\right) \rightarrow\left(\mathrm{u}_{1}, \ldots, \mathrm{u}_{\mathrm{i}}\right)$ as $\mathrm{m} \rightarrow \infty$ and $\mathrm{v}_{\mathrm{n}} \in \mathrm{X}_{\mathrm{n}}$ is fixed;

(2) $v_{n} \mapsto \sum_{n=1}^{i}\left\langle T_{n}\left(u_{1}, \ldots, u_{i}\right), \eta_{n}\left(u_{n}, v_{n}\right)\right\rangle_{X_{n}}$ is a convex $\forall u_{n} \in X_{n}$ 


\section{University of Thi-Qar Journal Vol.11 No.4 DEC 2016}

Web Site: https://jutq.utq.edu.iq/index.php/main Email: journal@jutq.utq.edu.iq

$\mathrm{H}_{\mathrm{H}}$ : $\limsup _{\mathrm{m}} \mathrm{H}_{\mathrm{i}}\left(\mathrm{u}_{1}^{\mathrm{m}}, \ldots, \mathrm{u}_{\mathrm{i}}^{\mathrm{m}}\right)=\mathrm{H}_{\mathrm{i}}\left(\mathrm{u}_{1}, \ldots, \mathrm{u}_{\mathrm{i}}\right) \quad$ whenever $\quad \mathrm{u}_{\mathrm{n}}^{\mathrm{m}} \rightarrow \mathrm{u}_{\mathrm{n}} \quad$ as $\mathrm{m} \rightarrow \infty$.

Theorem 3.1. Assume that the nonempty, bounded, closed and convex set $\mathrm{K}_{\mathrm{n}} \subset \mathrm{X}_{\mathrm{n}}$ for every $\mathrm{n}=\overline{1,1}$.If the conditions $\mathrm{H}_{\mathrm{F}}, \mathrm{H}_{\eta}, \mathrm{H}_{\mathrm{T}}$ and $\mathrm{H}_{\mathrm{H}}$ satisfy, then the system of nonlinear quasi- hemivariational inequalities (NQHZIS) admits at least one solution.

In what follows, we are going to present the following vector quasihemivariational inequality:

(VQHI) find $u \in K$,such that

$$
\left\langle\mathrm{T}_{\mathrm{n}} \eta(\mathrm{u}, \mathrm{v})\right\rangle+\mathrm{H}(\mathrm{u}) \mathrm{J}^{0}(\mathrm{Au} ; \mathrm{A \eta}(\mathrm{u}, \mathrm{v})) \geq\langle\mathrm{Fu}, \mathrm{v}-\mathrm{u}\rangle_{\mathrm{X}}
$$

For all $v \in \mathrm{K}$

Proposition 3.2. Assume that the assumptions $\mathrm{H}_{\mathrm{F}}, \mathrm{H}_{\eta}, \mathrm{H}_{\mathrm{T}}$ and $\mathrm{H}_{\mathrm{H}}$ satisfy, and that $\left(\mathrm{u}_{1}{ }^{0}, \ldots, \mathrm{u}_{\mathrm{i}}{ }^{0}\right) \in \mathrm{K}_{1} \times \ldots \times \mathrm{K}_{\mathrm{i}}$ is a solution of the inequality (VQHI), then $\mathrm{u}^{0}$ is a solution of the system (NQHI).

Proof. Indeed, if we fix a point $u_{n} \in K_{n}$ for every $n=\overline{1,1}$ and for $n \neq h$. We assume that $u_{h}=u_{h}{ }^{0}$. Therefore,from lemma 1.8 and $H_{\eta}(1)$,

$0 \leq\left\langle\mathrm{Tu}^{0}, \eta\left(\mathrm{u}^{0}, \mathrm{v}\right)\right\rangle_{\mathrm{X}}+\mathrm{H}\left(\mathrm{u}^{0}\right) \mathrm{J}^{0}\left(\mathrm{Au^{0 }} ; \mathrm{A \eta}\left(\mathrm{u}^{0}, \mathrm{v}\right)\right)-\left\langle\mathrm{Fu} \mathrm{u}^{0}, \mathrm{v}-\mathrm{u}^{0}\right\rangle_{\mathrm{X}}$

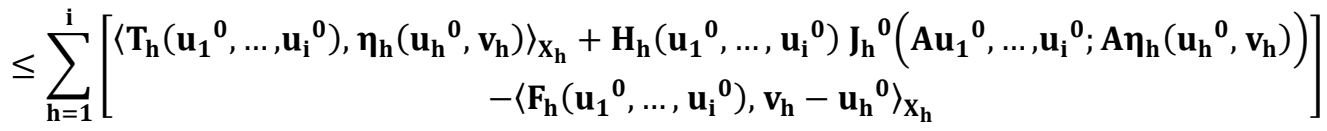

$=$

$\left\langle T_{n}\left(u_{1}^{0}, \ldots, u_{i}^{0}\right), \eta_{n}\left(u_{n}{ }^{0}, v_{n}\right)\right\rangle_{X_{n}}+$

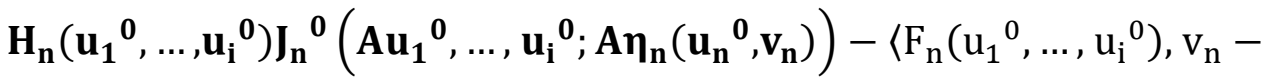
$\left.\mathrm{u}_{\mathrm{n}}{ }^{0}\right\rangle_{\mathrm{X}_{\mathrm{n}}}, \quad \forall \mathrm{n}=\overline{1.1}$.

This means that $\left(\mathrm{u}_{1}{ }^{0}, \ldots, \mathrm{u}_{\mathrm{i}}{ }^{0}\right) \in \mathrm{K}_{1} \times \ldots \times \mathrm{K}_{\mathrm{i}}$ is a solution of the inequality (NQHIS).

Remark 3.3. The mapping $v_{n} \mapsto J^{0}{ }_{, n}\left(\left(u_{1}, \ldots, u_{i}\right), \eta_{n}\left(u_{n}, v_{n}\right)\right)$ is a convex for every $\left(u_{1}, \ldots, u_{i}\right) \in E_{1} \times \ldots \times E_{i}$. It follows from the convexity of $\mathrm{J}^{0}{ }_{, \mathrm{n}}\left(\left(\mathrm{u}_{1}, \ldots, \mathrm{u}_{\mathrm{i}}\right), \mathrm{v}_{\mathrm{n}}\right)$ and linearity of $\eta_{\mathrm{n}}\left(\mathrm{u}_{\mathrm{n}}, \cdot\right)$ For every $\mathrm{n}=\overline{1,1}$. 


\section{University of Thi-Qar Journal Vol.11 No.4 DEC 2016}

Web Site: https://jutq.utq.edu.iq/index.php/main Email: journal@jutq.utq.edu.iq

Remark 3.4. Since $A$ is a linear compact operator we obtain that $A v_{n}$ converges strongly to some $A_{v} \in K$ and $u \in K, A \eta\left(v_{n}, u\right)$, converges strongly to $A \eta\left(v_{n}, u\right)$.

Applying this fact, together with Proposition 2.4(2), we get that

$$
\underset{n}{\lim \sup ^{0}}\left(A v_{n} ; A \eta\left(v_{n}, u\right)\right) \leq J^{0}(A v ; A \eta(u, v)) .
$$

In what follows, we are going to prove of Theorem 3.1.

Proof. According to Proposition 3.2, it is enough to prove that problem (VQHI) has at least one solution. We argue by contradiction, let us assume that (VQHI) has no solution. Then for each $\mathrm{u} \in \mathrm{K}$ there exists $\mathrm{v} \in \mathrm{K}$ such that

$$
\langle\mathrm{Tu}, \eta(\mathrm{u}, \mathrm{v})\rangle_{\mathrm{X}}+\mathrm{H}(\mathrm{u}) \mathrm{J}^{0}(\mathrm{Au} ; \mathrm{A \eta}(\mathrm{u}, \mathrm{v}))<\langle\mathrm{Fu}, \mathrm{v}-\mathrm{u}\rangle_{\mathrm{X}}
$$

Let as consider the set $\psi \subset \mathrm{K} \times \mathrm{K}$ as follows

$$
\begin{aligned}
& \psi=\left\{(\mathrm{u}, \mathrm{v}) \in \mathrm{K} \times \mathrm{K}:\langle\mathrm{Tu}, \eta(\mathrm{u}, \mathrm{v})\rangle_{\mathrm{X}}+\mathrm{H}(\mathrm{u}) \mathrm{J}^{0}(\mathrm{Au} ; \mathrm{A \eta}(\mathrm{u}, \mathrm{v}))\right. \\
\geq & \left.\langle\mathrm{Fu}, \mathrm{v}-\mathrm{u}\rangle_{\mathrm{X}}\right\} . \quad
\end{aligned}
$$

We shall prove $\psi$ satisfies the conditions of Tarafdar Lemma for weak topology of the space $\mathrm{X}$. Let $\alpha: \mathrm{K} \multimap \mathrm{K}$ defined by

$$
\alpha(\mathrm{u})=\{\mathrm{v} \in \mathrm{K}:(\mathrm{v}, \mathrm{u}) \notin \psi\} .
$$

Claim 1: $\alpha(\mathrm{u})$ is nonempty and convex $\forall \mathrm{u} \in \mathrm{K}$.

Obviously, $\alpha(\mathrm{u})$ is a nonempty for each $\mathrm{u} \in \mathrm{K}$. Let us choose $\mathrm{v}^{\prime}, \mathrm{v}^{\prime \prime} \in$ $\alpha(\mathrm{u}), \mathrm{t} \in(0,1)$ and $\mathrm{v}^{\mathrm{t}}=\mathrm{tv}^{\prime}+(1-\mathrm{t}) \mathrm{v}^{\prime \prime}$. Using $\mathrm{H}_{\mathrm{T}}(2)$, one can have 


\section{University of Thi-Qar Journal Vol.11 No.4 DEC 2016}

Web Site: https://jutq.utq.edu.iq/index.php/main Email: journal@jutq.utq.edu.iq

$$
\begin{aligned}
& \left\langle T(u), \eta\left(u, v^{t}\right)\right\rangle_{X}=\sum_{n=1}^{i}\left\langle T_{n}\left(u_{1}, \ldots, u_{i}\right), \eta_{n}\left(u_{n}, t_{n}{ }^{\prime}+(1-t) v_{n}{ }^{\prime \prime}\right)\right\rangle_{X_{n}} \\
& \leq t \sum_{n=1}^{i}\left\langle T_{n}\left(u_{1}, \ldots, u_{i}\right), \eta_{n}\left(u_{n}, v_{n}\right)\right\rangle_{X_{n}} \\
& +(1-t) \sum_{n=1}^{i}\left\langle T_{n}\left(u_{1}, \ldots, u_{i}\right), \eta_{n}\left(u_{n}, v_{n}\right)\right\rangle_{X_{n}} \\
& =t\left\langle T(u), \eta\left(u, v^{\prime}\right)\right\rangle_{X_{n}}+(1-t)\left\langle T(u), \eta\left(u, v^{\prime \prime}\right)\right\rangle_{X_{n}}
\end{aligned}
$$

$\forall \mathrm{t} \in(0,1)$, so $\mathrm{v} \mapsto\left\langle\mathrm{T}(\mathrm{u}), \eta\left(\mathrm{u}, \mathrm{v}^{\mathrm{t}}\right)\right\rangle_{\mathrm{X}}$ is convex. On the other hand, Remark $(1,8)$ and continuity of $\mathrm{H}$, we deduce that the mapping $\mathrm{v} \mapsto \mathrm{H}(\mathrm{u}) \mathrm{J}^{0}(\mathrm{Au} ; \mathrm{A \eta}(\mathrm{u}, \mathrm{v}))$ is convex. Applying the fact that the mapping $\mathrm{v} \mapsto\langle\mathrm{F}(\mathrm{u}), \mathrm{v}-\mathrm{u}\rangle_{\mathrm{X}}$ is an affine, then $\alpha(\mathrm{u})$ is a convex for a fixed $\mathrm{u} \in \mathrm{K}$.

Claim 2. $\left[\alpha^{-1}(v)\right]^{c}=\{u \in K:(v, u) \in \psi\}$ is weakly set in $K$.

It is enough to prove that for any fixed point $v \in K$, the function $G$ : $K \rightarrow R$,

$\mathrm{G}(\mathrm{u})=$

$\langle T(u), \eta(u, v)\rangle_{X}+H(u) J^{0}(A u ; A \eta(u, v))-\langle F(u), v-u\rangle_{X}$

is weakly upper semi continuous. This is equivalent to say that mappings:

$\langle T(u), \eta(u, v)\rangle, H(u) J^{0}(A u ; A \eta(u, v))$ is weakly upper semi continuous while the mapping $\langle F(u), v-u\rangle_{X}$ is weakly lower semi continuous for the fixed $v \in K$. Assume that $\left\{\mathrm{u}^{\mathrm{m}}\right\} \subset \mathrm{K}$ be a sequence such that $\mathrm{u}^{\mathrm{m}} \rightarrow \mathrm{u} \in \mathrm{K}$ as $\mathrm{m} \rightarrow \infty$. From $\mathrm{H}_{\eta}(3)$ and $\mathrm{H}_{\mathrm{T}}$ for every $\mathrm{n}=\overline{1,1}$, then

$$
\begin{aligned}
& \underset{m}{\limsup }\left\langle T\left(u^{m}\right) \eta\left(u^{m}, v\right)\right\rangle_{X}=\underset{m}{\limsup } \sum_{n=1}^{i}\left\langle T_{n}\left(u_{1}{ }^{m}, \ldots, u_{i}{ }^{m}\right), \eta_{n}\left(u_{n}{ }^{m}, v_{n}\right)\right\rangle_{X_{n}} \\
& \leq \sum_{\mathrm{n}=1}^{\mathrm{i}} \limsup _{\mathrm{m}}\left\langle\mathrm{T}_{\mathrm{n}}\left(\mathrm{u}_{1}{ }^{\mathrm{m}}, \ldots, \mathrm{u}_{\mathrm{i}}^{\mathrm{m}}\right), \eta_{\mathrm{n}}\left(\mathrm{u}_{\mathrm{n}}^{\mathrm{m}}, \mathrm{v}_{\mathrm{n}}\right)\right\rangle_{\mathrm{X}_{\mathrm{n}}}
\end{aligned}
$$




\section{University of Thi-Qar Journal Vol.11 No.4 DEC 2016}

Web Site: https://jutq.utq.edu.iq/index.php/main Email: journal@jutq.utq.edu.iq

$$
\begin{aligned}
& \leq \sum_{n=1}^{\mathrm{i}}\left\langle\mathrm{T}_{\mathrm{n}}\left(\mathrm{u}_{1}, \ldots, \mathrm{u}_{\mathrm{i}}\right), \eta_{\mathrm{n}}\left(\mathrm{u}_{\mathrm{n}}, \mathrm{v}_{\mathrm{n}}\right)\right\rangle_{\mathrm{X}_{\mathrm{n}}} \\
& =\langle\mathrm{T}(\mathrm{u}), \eta(\mathrm{u}, \mathrm{v})\rangle_{\mathrm{X}}
\end{aligned}
$$

This means that the mapping $\mathrm{u} \longmapsto\langle\mathrm{Tu}, \eta(\mathrm{u}, \mathrm{v})\rangle$ is weakly u.s.c.

One the other hand, from Remark $3.4, \mathrm{H}_{\mathrm{H}}$ and $\mathrm{J}^{0}(\mathrm{u}, \mathrm{v})$ is u.s.c. Then

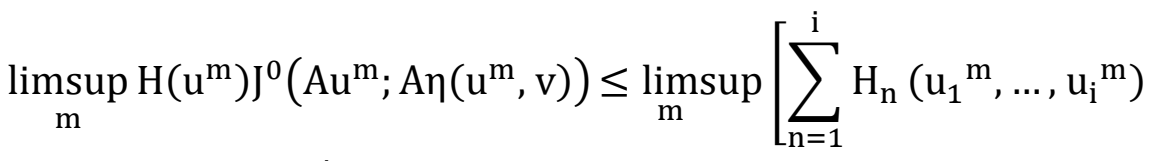

$$
\begin{aligned}
& \left.-\sum_{n=1}^{\mathrm{i}} \mathrm{H}_{\mathrm{n}}\left(\mathrm{u}_{1}, \ldots, \mathrm{u}_{\mathrm{i}}\right)\right] \mathrm{J}^{0}\left(\mathrm{Au}^{\mathrm{m}} ; \operatorname{A\eta }\left(\mathrm{u}_{\mathrm{i}}^{\mathrm{m}}, \mathrm{v}_{\mathrm{i}}\right)\right) \\
& +\limsup _{\mathrm{m}} \sum_{\mathrm{n}=1}^{\mathrm{i}} \mathrm{H}_{\mathrm{n}}\left(\mathrm{u}_{1}, \ldots, \mathrm{u}_{\mathrm{i}}\right) \mathrm{J}^{0}\left(A \mathrm{u}^{\mathrm{m}} ; \operatorname{A\eta }\left(\mathrm{u}_{\mathrm{i}}^{\mathrm{m}}, \mathrm{v}_{\mathrm{i}}\right)\right) \\
& \leq 0+\limsup _{\mathrm{m}} \sum_{\mathrm{n}=1}^{\mathrm{i}} \mathrm{H}_{\mathrm{n}}\left(\mathrm{u}_{1}, \ldots, \mathrm{u}_{\mathrm{i}}\right) \mathrm{J}^{0}\left(A u^{\mathrm{m}} ; \operatorname{A\eta }\left(\mathrm{u}_{\mathrm{i}}^{\mathrm{m}}, \mathrm{v}_{\mathrm{i}}\right)\right) \\
& =H(u) J^{0}(A u ; A \eta(u, v)) \text {. }
\end{aligned}
$$

Finally, by applying $\mathrm{H}_{\mathrm{F}}$, one can get

$$
\begin{aligned}
\langle F u, v-u\rangle_{X} & =\sum_{n=1}^{i}\left\langle F n\left(u_{1}, \ldots, u_{i}\right), v_{n}-u_{n}\right\rangle_{X_{n}} \\
& \leq \liminf _{m} \sum_{n=1}^{i}\left\langle F n\left(u_{1}^{m}, \ldots, u_{i}{ }^{m}\right), v_{n}-u_{n}{ }^{m}\right\rangle_{X_{n}} \\
& =\liminf _{m}\left\langle F\left(u^{m}\right), v-u^{m}\right\rangle_{X_{n}} .
\end{aligned}
$$

Therefore, $G$ is weakly u.s.c. Hence, the set $\{u \in K: G(u) \geq \beta\}$ is weakly closed for every $\beta \in \mathrm{R}$. Taking $\beta=0$,we get that $\left[\alpha^{-1}(v)\right]^{\mathrm{c}}$ is weakly closed.

Claim 3: $\mathrm{K}=\mathrm{U} \alpha^{-1}(\mathrm{v})$. 


\section{University of Thi-Qar Journal Vol.11 No.4 DEC 2016}

Web Site: https://jutq.utq.edu.iq/index.php/main Email: journal@jutq.utq.edu.iq

It is sufficing to prove that $\mathrm{K} \subset \mathrm{U} \alpha^{-1}(\mathrm{v})$. Let $\mathrm{u} \in \mathrm{K}$,so by 3.3, there exists $\mathrm{v} \in \mathrm{K}$ such that $\mathrm{v} \in \alpha(\mathrm{u})$, this means that $\mathrm{u} \in \alpha^{-1}(\mathrm{v})$. Therefore, $\mathrm{K} \subset$ $U\left(\alpha^{-1}(v)\right)$.

Claim 4: $S=\bigcap_{\mathrm{v} \in \mathrm{K}}\left[\alpha^{-1}(\mathrm{v})\right]^{\mathrm{c}}$ is empty or weakly compact.

Since $\left[\alpha^{-1}(v)\right]^{c}$ is weakly closed on a nonempty, bounded, closed and convex set $\mathrm{K}$ of reflexive Banach space $\mathrm{X}$, then $\mathrm{K}$ is weakly compact set. Hence, $S$ is weakly compact set as it an intersection of weakly closed subsets of weakly compact. Therefore.by conditions $\left(\mathrm{T}_{1}-\mathrm{T}_{2}\right)$ of Lemma Tarafrar, there exists $u^{0} \in \alpha\left(u^{0}\right)$ which implies

$$
0=\left\langle\mathrm{Tu}^{0}, \eta\left(\mathrm{u}^{0}, \mathrm{v}\right)\right\rangle+\mathrm{H}\left(\mathrm{u}^{0}\right) \mathrm{J}^{0}\left(\mathrm{Au}^{0} ; \mathrm{A \eta}\left(\mathrm{u}^{0}, \mathrm{v}\right)\right)-\left\langle\mathrm{Fu}^{0}, \mathrm{v}-\mathrm{u}^{0}\right\rangle_{\mathrm{X}}<0 .
$$

So, we have reached a contradiction. Therefore, (NQHIS) admits at least one solution.

$H_{S}:$ let $\left(u^{1}, \ldots, u^{i}\right),\left(v^{1}, \ldots, v^{i}\right) \in X_{1} \times \ldots \times X_{i}$ for every $n=\overline{1, l}$.

(i) $\quad \eta_{\mathrm{n}}\left(\mathrm{u}_{\mathrm{n}}, \mathrm{v}_{\mathrm{n}}\right)+\eta_{\mathrm{n}}\left(\mathrm{v}_{\mathrm{n}}, \mathrm{u}_{\mathrm{n}}\right)=0 \forall \mathrm{u}_{\mathrm{n}} \in \mathrm{X}_{\mathrm{n}}$;

(ii) $\left\langle\mathrm{T}_{\mathrm{n}}\left(\mathrm{u}_{1}, \ldots, \mathrm{u}_{\mathrm{i}}\right), \eta_{\mathrm{n}}\left(\mathrm{u}_{\mathrm{n}}, \mathrm{v}_{\mathrm{n}}\right)\right\rangle_{\mathrm{x}_{\mathrm{n}}}+\left\langle\mathrm{T}_{\mathrm{n}}\left(\mathrm{v}_{1} \ldots, \mathrm{v}_{\mathrm{i}}\right), \eta_{\mathrm{n}}\left(\mathrm{v}_{\mathrm{n}}, \mathrm{u}_{\mathrm{n}}\right)\right\rangle_{\mathrm{x}_{\mathrm{n}}} \geq 0$;

(iii) $\quad v_{K} \mapsto \sum_{n=1}^{i}\left\langle T_{n}\left(u_{1}, \ldots, u_{i}\right), \eta_{n}\left(u_{n}, v_{n}\right)\right\rangle_{X_{n}}$ is weakly 1.s.c;

(iv) For each $v_{i} \in X_{i}$, the mapping $\mathrm{v} \mapsto \sum_{\mathrm{n}=1}^{\mathrm{i}}\left\langle\mathrm{T}_{\mathrm{n}}\left(\mathrm{u}_{1}, \ldots, \mathrm{u}_{\mathrm{i}}\right), \eta_{\mathrm{n}}\left(\mathrm{u}_{\mathrm{n}}, \mathrm{v}_{\mathrm{n}}\right)\right\rangle_{\mathrm{X}_{\mathrm{n}}}$ is concave.

lemma 3.5. suppose that $\mathrm{H}_{\mathrm{S}}$ holds. Then the following hold

(i) $\mathrm{T}$ is $\eta-$ monotone operator.

(ii) The mapping $\mathrm{u} \mapsto\langle\mathrm{Tu}, \eta(\mathrm{u}, \mathrm{v})\rangle_{\mathrm{X}}$ is weakly u.s.c $\forall \mathrm{v} \in \mathrm{X}$.

(iii) $\mathrm{v}_{\mathrm{k}} \mapsto \sum_{\mathrm{n}=1}^{\mathrm{i}}\left\langle\mathrm{T}_{\mathrm{n}}\left(\mathrm{u}_{1}, \ldots, \mathrm{u}_{\mathrm{i}}\right), \eta_{\mathrm{n}}\left(\mathrm{u}_{\mathrm{n}}, \mathrm{v}_{\mathrm{n}}\right)\right\rangle_{\mathrm{X}_{\mathrm{n}}}$ is weakly l.s.c;

(iv) For each $v_{i} \in X_{i}$ the mapping $\mathrm{v} \longmapsto \sum_{\mathrm{n}=1}^{\mathrm{i}}\left\langle\mathrm{T}_{\mathrm{n}}\left(\mathrm{u}_{1}, \ldots, \mathrm{u}_{\mathrm{i}}\right), \eta_{\mathrm{n}}\left(\mathrm{u}_{\mathrm{n}}, \mathrm{v}_{\mathrm{n}}\right)\right\rangle_{\mathrm{X}_{\mathrm{n}}}$ is concave.

Lemma 3.5. suppose that $\mathrm{H}_{\mathrm{S}}$ holds. Then the following hold

(i) $\mathrm{T}$ is $\eta$-monotone operator.

(ii) The mapping $\mathrm{u} \mapsto\langle T u, \eta(\mathrm{u}, \mathrm{v})\rangle_{\mathrm{X}}$ is weakly u.s.c $\forall \mathrm{v} \in \mathrm{X}$.

(iii) The mapping $\mathrm{v} \mapsto\langle\mathrm{Tu} \text {; } \eta(\mathrm{u}, \mathrm{v})\rangle_{\mathrm{X}}$ is convex $\forall \mathrm{u} \in \mathrm{X}$. 


\section{University of Thi-Qar Journal Vol.11 No.4 DEC 2016}

Web Site: https://jutq.utq.edu.iq/index.php/main Email: journal@jutq.utq.edu.iq

Proof. From $\mathrm{H}_{\mathrm{S}}(\mathrm{i}-\mathrm{ii})$, we have

$$
\begin{aligned}
\langle\mathrm{Tu}-\mathrm{Tv}, \eta(\mathrm{u}, \mathrm{v})\rangle_{\mathrm{X}} & =\langle\mathrm{Tu}, \eta(\mathrm{u}, \mathrm{v})\rangle_{\mathrm{X}}-\langle\mathrm{Tv}, \eta(\mathrm{u}, \mathrm{v})\rangle_{\mathrm{X}} \\
& =\sum_{\mathrm{n}=1}^{\mathrm{i}}\left\langle\mathrm{T}_{\mathrm{n}}\left(\mathrm{u}_{1}, \ldots, \mathrm{u}_{\mathrm{i}}\right), \eta_{\mathrm{n}}\left(\mathrm{u}_{\mathrm{n}}, \mathrm{v}_{\mathrm{n}}\right)\right\rangle_{\mathrm{X}_{\mathrm{n}}} \\
& -\sum_{\mathrm{n}=1}^{\mathrm{i}}\left\langle\mathrm{T}_{\mathrm{n}}\left(\mathrm{v}_{1}, \ldots, \mathrm{v}_{\mathrm{i}}\right), \eta_{\mathrm{n}}\left(\mathrm{u}_{\mathrm{n}}, \mathrm{v}_{\mathrm{n}}\right)\right\rangle_{\mathrm{X}_{\mathrm{n}}} \\
& =\sum_{\mathrm{n}=1}^{\mathrm{i}}\left\langle\mathrm{T}_{\mathrm{n}}\left(\mathrm{u}_{1}, \ldots, \mathrm{u}_{\mathrm{i}}\right), \eta_{\mathrm{n}}\left(\mathrm{u}_{\mathrm{n}}, \mathrm{v}_{\mathrm{n}}\right)\right\rangle_{\mathrm{X}_{\mathrm{n}}} \\
& +\sum_{\mathrm{n}=1}^{\mathrm{i}}\left\langle\mathrm{T}_{\mathrm{n}}\left(\mathrm{v}_{1}, \ldots, \mathrm{v}_{\mathrm{i}}\right), \eta_{\mathrm{n}}\left(\mathrm{v}_{\mathrm{n}}, \mathrm{u}_{\mathrm{n}}\right)\right\rangle_{\mathrm{X}_{\mathrm{n}}} \\
& \geq 0 .
\end{aligned}
$$

Prove second assertion, let $\left\{\mathrm{u}^{\mathrm{m}}\right\} \subset \mathrm{X}$ be a sequence which converges weakly to some $\mathrm{u} \in \mathrm{X}$. According to $\mathrm{H}_{\mathrm{S}}(\mathrm{i}-\mathrm{ii})$ and the verity that $\mathrm{u}^{\mathrm{m}} \rightarrow \mathrm{u}$, one can obtain

$$
\begin{aligned}
& \underset{m}{\limsup }\left\langle T\left(u^{m}\right), \eta\left(u^{m}, v\right)\right\rangle_{X}=\limsup _{m} \sum_{n=1}^{i}\left\langle T_{n}\left(u_{1}{ }^{m}, \ldots, u_{i}^{m}\right), \eta_{n}\left(u_{n}{ }^{m}, v_{n}\right)\right\rangle_{X_{n}} \\
& -\liminf _{m} \sum_{n=1}^{i}\left\langle T_{n}\left(u_{1}{ }^{m}, \ldots, u_{i}{ }^{m}\right), \eta_{n}\left(v_{n}, u_{n}{ }^{m}\right)\right\rangle_{X_{n}} \\
& =-\sum_{n=1}^{i} \liminf _{m}\left\langle T_{n}\left(u_{1}^{m}, \ldots, u_{i}^{m}\right), \eta_{n}\left(v_{n}, u_{n}^{m}\right)\right\rangle_{X_{n}} \\
& \leq-\sum_{n=1}^{i}\left\langle T_{n}\left(u_{1}, \ldots, u_{i}\right), \eta_{n}\left(v_{n}, u_{n}\right)\right\rangle_{X_{n}} \\
& =\sum_{n=1}^{i}\left\langle T_{n}\left(u_{1}, \ldots, u_{i}\right), \eta_{n}\left(u_{n}, v_{n}\right)\right\rangle_{X_{n}}
\end{aligned}
$$




\section{University of Thi-Qar Journal Vol.11 No.4 DEC 2016}

Web Site: https://jutq.utq.edu.iq/index.php/main Email: journal@jutq.utq.edu.iq

$$
=\langle\mathrm{Tu}, \eta(\mathrm{u}, \mathrm{v})\rangle_{\mathrm{X}}
$$

Prove third assertion, let $\mathrm{u}, \mathrm{v}^{\prime}, \mathrm{v}^{\prime \prime} \in \mathrm{X}$ then, by $\mathrm{H}_{\mathrm{S}}(\mathrm{i}-\mathrm{iv}) \operatorname{such}$ that $\mathrm{v}^{\mathrm{t}}=$ $\mathrm{tv}^{\prime}+(1-\mathrm{t}) \mathrm{v}^{\prime \prime} \quad \forall \mathrm{t} \in(0,1)$, then

$$
\begin{aligned}
\langle T u, \eta(u, v)\rangle_{X}= & \sum_{n=1}^{i}\left\langle T_{n}\left(u_{1}, \ldots, u_{i}\right), \eta_{n}\left(u_{n}, t_{n}{ }^{\prime}(1-t) v_{n}{ }^{\prime \prime}\right)\right\rangle_{X_{n}} \\
& -\sum_{n=1}^{i}\left\langle T_{n}\left(u_{1}, \ldots, u_{i}\right), \eta_{n}\left(t v_{n}{ }^{\prime}(1-t) v_{n}{ }^{\prime \prime}, u_{n}\right)\right\rangle_{X_{n}} \\
\leq & -\sum_{n=1}^{i} t\left\langle T_{n}\left(u_{1}, \ldots, u_{i}\right), \eta_{n}\left(v_{n}{ }^{\prime}, u_{n}\right\rangle_{x_{n}}\right. \\
& -\sum_{n=1}^{i}(1-t)\left\langle T_{n}\left(u_{1}, \ldots, u_{i}\right), \eta_{n}\left(v_{n}{ }^{\prime \prime}, u_{n}\right\rangle_{X_{n}}\right. \\
= & \sum_{n=1}^{i} t\left\langle T_{n}\left(u_{1}, \ldots, u_{i}\right), \eta_{n}\left(u_{n}, v_{n}{ }^{\prime}\right)\right\rangle_{X_{n}} \\
+ & \sum_{n=1}^{i}(1-t)\left\langle T_{n}\left(u_{1}, \ldots, u_{i}\right), \eta_{n}\left(u_{n}, v_{n}{ }^{\prime \prime}\right)\right\rangle_{X_{n}} \\
+ &
\end{aligned}
$$

Theorem 3.6. Assume that the nonempty, bounded, closed and convex set $K_{n} \subset X_{n}$ for every $n=\overline{1,1}$. If the condition $H_{F}, H_{\eta}, H_{S}$ and $H_{H}$ satisfy, then the system of nonlinear quasi- hemivariational inequalities (NQHIS) admits at least one solution.

Proof. Suppose that the set $\psi \subset \mathrm{K} \times \mathrm{K}$ defined as follows:

$$
\begin{aligned}
\Psi=\{(\mathrm{u}, \mathrm{v}) \in & \mathrm{K} \times \mathrm{K}:\langle\mathrm{Tv}, \eta(\mathrm{u}, \mathrm{v})\rangle_{\mathrm{X}}+\mathrm{H}(\mathrm{u}) \mathrm{J}^{0}(\mathrm{Au} ; \mathrm{A \eta}(\mathrm{u}, \mathrm{v}))-\langle\mathrm{Fu}, \mathrm{v}-\mathrm{u}\rangle_{\mathrm{X}} \\
& \geq 0\} .
\end{aligned}
$$




\section{University of Thi-Qar Journal Vol.11 No.4 DEC 2016}

Web Site: https://jutq.utq.edu.iq/index.php/main Email: journal@jutq.utq.edu.iq

One can follow the same steps in the Theorem 3.1 to conclude that the conditions required in Tarafdar fixed point Theorem are hold. Therefore, there exists $\mathrm{u}_{0} \in \mathrm{K}$ such that

$$
\left\langle\operatorname{Tv}, \eta\left(u_{0}, v\right)\right\rangle_{X}+H\left(u_{0}\right) J^{0}\left(A u_{0} ; A \eta\left(u_{0}, v\right)\right) \geq\left\langle\mathrm{Fu}_{0}, v-u_{0}\right\rangle_{X} .
$$

Since $\mathrm{T}$ is $\eta$-monotone operator, so

$$
\langle\mathrm{Tu}-\mathrm{Tv}, \eta(\mathrm{u}, \mathrm{v})\rangle_{\mathrm{X}} \geq 0 .
$$

Adding (3.7) and (3.8) we have $\mathrm{u}_{0}$ solves (VQHVI), which applying Proposition.

In order to highlight the advantage of our inequality problem dedicate the last section to two applications, one in equilibrium problem and the other in an abstract nonlinear quasi-hemivariational inequality.

\section{APPLICATIONS}

\subsection{First application}

In this subsection, we present a result one the connection between equilibrium problems and our inequalities in the case $\mathrm{T}$ is set-valued. It is clear any solution of the quasi-hemivariational inequality is a solution of the equilibrium problem, where the equilibrium bifunction $\Lambda(\mathrm{u}, \mathrm{v}): \mathrm{K} \times \mathrm{K} \rightarrow \mathrm{R}$ is defined by

$$
\begin{gathered}
\Lambda(\mathrm{u}, \mathrm{v})=\sup _{\mathrm{z} \in \mathrm{T}(\mathrm{u})}\langle\mathrm{z}, \eta(\mathrm{u}, \mathrm{v})\rangle_{\mathrm{X}}+\mathrm{H}(\mathrm{u}) \mathrm{J}^{0}(\mathrm{Au} ; \mathrm{A \eta}(\mathrm{u}, \mathrm{v})) \\
-\langle\mathrm{Fu}, \mathrm{v}-\mathrm{u}\rangle_{\mathrm{X}} .
\end{gathered}
$$

$\mathrm{u}, \mathrm{v} \in \mathrm{K}$.

In what following, let rewrite (3.3) where T is set- valued:

Find $\mathrm{u} \in \mathrm{K}$ and $\mathrm{z} \in \mathrm{T}(\mathrm{u})$ such that

$$
\langle\mathrm{z}, \eta(\mathrm{u}, \mathrm{v})\rangle_{\mathrm{X}}+\mathrm{H}(\mathrm{u}) \mathrm{J}^{0}(\mathrm{Au} ; \mathrm{A \eta}(\mathrm{u}, \mathrm{v})) \geq\langle\mathrm{Fu}, \mathrm{v}-\mathrm{u}\rangle_{\mathrm{X}}
$$

$\forall \mathrm{v} \in \mathrm{K}$.

The next result was introduced by B. Allechea, V. Radulescu and M. Sebaouia in [2]. We extend this result.

Corollary 4.1. If a set-valued $\mathrm{T}: \mathrm{X} \multimap \mathrm{X}^{*}$ has a nonempty,convex and weak $^{*}$ compact values, then any solution of the equilibrium problem (EP) is a solution of the problem (4.2). 


\section{University of Thi-Qar Journal Vol.11 No.4 DEC 2016}

Web Site: https://jutq.utq.edu.iq/index.php/main Email: journal@jutq.utq.edu.iq

Proof. Let $\mathrm{u}^{*} \in \mathrm{K} \subset \mathrm{X}$ such that $\Lambda\left(\mathrm{u}^{*}, \mathrm{v}\right) \geq 0$ for every $\mathrm{v} \in \mathrm{K}$. Arguing by contradiction. Let us assume that problem (4.2) has no solutions. So, there exists $\mathrm{v}_{\mathrm{z}} \in \mathrm{K}$ for any $\mathrm{z} \in \mathrm{T}\left(\mathrm{u}^{*}\right)$ in which

$\left\langle\mathrm{z}, \eta\left(\mathrm{u}^{*}, \mathrm{v}_{\mathrm{z}}\right)\right\rangle_{\mathrm{X}}+\mathrm{H}\left(\mathrm{u}^{*}\right) \mathrm{J}^{0}\left(\mathrm{Au} \mathrm{u}^{*} ; \mathrm{A \eta}\left(\mathrm{u}^{*}, \mathrm{v}_{\mathrm{z}}\right)\right)-\left\langle\mathrm{Fu}^{*}, \mathrm{v}_{\mathrm{z}}-\mathrm{u}^{*}\right\rangle_{\mathrm{X}}<0$.

Define P: $\mathrm{X}^{*} \times \mathrm{K} \times \mathrm{K} \rightarrow \mathrm{R}$ such that

$$
\mathrm{P}\left(\mathrm{z}, \mathrm{v}, \mathrm{u}^{*}\right):=\left\langle\mathrm{z}, \eta\left(\mathrm{u}^{*}, \mathrm{v}\right)\right\rangle_{\mathrm{X}}+\mathrm{H}\left(\mathrm{u}^{*}\right) \mathrm{J}^{0}\left(\mathrm{Au}^{*} ; \mathrm{A \eta}\left(\mathrm{v}, \mathrm{u}^{*}\right)\right)-\left\langle\mathrm{Fu}^{*}, \mathrm{v}-\mathrm{u}^{*}\right\rangle_{\mathrm{X}}
$$

One can easily show that for any $v \in K$, the mapping defined on $X^{*}$ by

$$
\mathrm{z} \mapsto \mathrm{P}\left(\mathrm{z}, \mathrm{v}, \mathrm{u}^{*}\right)
$$

Is weak ${ }^{*}$-continuous. Let $\mathrm{S}:=-\mathrm{P}$ so, $\mathrm{S}$ is weak $^{*}$-continuous with respect to first variable where $S\left(\mathrm{z}, \mathrm{v}, \mathrm{u}^{*}\right)>0$. Define $\Upsilon_{\mathrm{v}, \mathrm{u}^{*}}: X \rightarrow \mathrm{R}$ such that $\Upsilon_{\mathrm{v}, \mathrm{u}^{*}}(\mathrm{z}):=$ $\mathrm{S}\left(\mathrm{z}, \mathrm{v}, \mathrm{u}^{*}\right)$ is weak $^{*}$-continuous too, So, $\forall \mathrm{z} \in \mathrm{T}\left(\mathrm{u}^{*}\right)$ there exists $\mathrm{v}_{\mathrm{z}} \in \mathrm{K}$ such that $\mathrm{x} \in \mathrm{U}_{\mathrm{v} \in \mathrm{K}, \rho>0}\left\{\mathrm{w}: \mathrm{S}\left(\mathrm{z}, \mathrm{v}, \mathrm{u}^{*}\right)>\rho\right\}=U_{\mathrm{v} \in \mathrm{K}, \rho>0} \Upsilon_{\mathrm{v}, \mathrm{u}^{*}}{ }^{-1}(\rho,+\infty)$ is weak $^{*}-$ open. Which shows

$$
\mathrm{T}\left(\mathrm{u}^{*}\right) \subset \bigcup_{\mathrm{v} \in \mathrm{K}} \Upsilon_{\mathrm{v}, \mathrm{u}^{*}}{ }^{-1}(\rho,+\infty) .
$$

Since $\mathrm{T}\left(\mathrm{u}^{*}\right)$ is weak ${ }^{*}$-compact, there is a finite sub cover

$$
\begin{aligned}
\Upsilon_{\mathrm{v}_{1}, \mathrm{u}^{*}}{ }^{-1}\left(\rho_{1},+\infty\right), \ldots, \Upsilon_{\mathrm{v}_{\mathrm{n} 1}, \mathrm{u}^{*}}\left(\rho_{\mathrm{n}},+\infty\right), \text { i.e., } \\
\mathrm{T}\left(\mathrm{u}^{*}\right) \subset \bigcup_{\mathrm{i}=1}^{\mathrm{n}} \Upsilon_{\mathrm{v}_{\mathrm{i}}, \mathrm{u}^{*}}{ }^{-1}\left(\rho_{\mathrm{i}},+\infty\right) .
\end{aligned}
$$

Letting $\rho=\min \left\{\rho_{i}, \ldots, \rho_{n}\right\}$. So,

$$
\mathrm{T}\left(\mathrm{u}^{*}\right) \subset \bigcup_{\mathrm{i}=1}^{\mathrm{n}} \Upsilon_{\mathrm{v}_{\mathrm{i}}, \mathrm{u}^{*}}{ }^{-1}(\rho,+\infty) \text {. }
$$

For every $\mathrm{z} \in \mathrm{T}\left(\mathrm{u}^{*}\right)$ then

$$
\operatorname{Max}_{\mathrm{n}=1,1} \mathrm{~S}\left(\mathrm{z}, \mathrm{v}, \mathrm{u}^{*}\right)>\rho
$$

It means that

$$
\operatorname{Min}_{\mathrm{n}=1, \mathrm{l}} \mathrm{S}\left(\mathrm{z}, \mathrm{v}, \mathrm{u}^{*}\right)<-\rho .
$$

For every $\mathrm{z} \in \mathrm{T}\left(\mathrm{u}^{*}\right)$. One can easily show that

$$
\mathrm{z} \mapsto \mathrm{P}\left(\mathrm{z}, \mathrm{v}_{\mathrm{i}}, \mathrm{u}^{*}\right)
$$

is convex and proper with domain containing $\mathrm{T}\left(\mathrm{u}^{*}\right)$. Then by Theorem 21.1 in [23] there exists $\lambda \geq 0$, such that $\sum_{\mathrm{i}=1}^{\mathrm{n}} \lambda_{\mathrm{i}}=1$ for every $\mathrm{n}=\overline{1,1}$. So, $\sum_{\mathrm{i}=1}^{\mathrm{n}} \lambda_{\mathrm{i}} \mathrm{P}\left(\mathrm{z}, \mathrm{v}_{\mathrm{i}}, \mathrm{u}^{*}\right)<-\rho$. It means that 


\section{University of Thi-Qar Journal Vol.11 No.4 DEC 2016}

Web Site: https://jutq.utq.edu.iq/index.php/main Email: journal@jutq.utq.edu.iq

$$
\sum_{i=1}^{n} \lambda_{i}\left[\left\langle z, \eta\left(u^{*}, v_{i}\right)\right\rangle_{X}+H\left(u^{*}\right) J^{0}\left(A u^{*} ; A \eta\left(u^{*}, v_{i}\right)\right)-\left\langle F u^{*}, v_{i}-u^{*}\right\rangle_{X}\right]<-\rho .
$$

Set $v^{*}=\sum_{\mathrm{i}=1}^{\mathrm{n}} \lambda_{\mathrm{i}} \mathrm{v}_{\mathrm{i}}$. Therefore, by use remark (3.3) and remark (3.4) we have

$$
\left\langle\mathrm{z}, \eta\left(\mathrm{u}^{*}, \mathrm{v}^{*}\right\rangle_{\mathrm{X}}+\mathrm{H}\left(\mathrm{u}^{*}\right) \mathrm{J}^{0}\left(\mathrm{Au} \mathrm{u}^{*} ; \mathrm{A \eta}\left(\mathrm{u}^{*}, \mathrm{v}^{*}\right)\right)-\left\langle\mathrm{Fu}^{*}, \mathrm{v}^{*}-\mathrm{u}^{*}\right\rangle_{\mathrm{X}}<-\rho\right.
$$

for every $\mathrm{z} \in \mathrm{T}\left(\mathrm{u}^{*}\right)$, which implies that $\Lambda\left(\mathrm{u}^{*}, \mathrm{v}^{*}\right)<0$ is a contradiction.

\subsection{Second application.}

In this subsection, we are going to apply our main result, expressed in the previous section for systems of an abstract nonlinear quasi- hemivariational inequality. We assume that $\Omega$ is a bounded, open subset of $\mathbb{R}^{\mathbb{N}}$. j: $\Omega \times \mathrm{R}^{\mathrm{N}} \times$ $\ldots \times \mathrm{R}^{\mathrm{N}} \rightarrow \mathbb{R}$ is a Carathe'odory function and that $\mathrm{j}(\mathrm{z}, \ldots, \cdot)$ is a locally Lipchitz for every $z \in \Omega$ and the following conditions hold:

$H_{j}$ : there exists $a_{n} \in L^{\frac{p}{p-1}}\left(\Omega, \mathbb{R}_{+}\right)$in which

$$
\left|\mathrm{w}_{\mathrm{n}}\right| \leq \mathrm{a}_{\mathrm{n}}(\mathrm{x})+\mathrm{b}_{\mathrm{n}}(\mathrm{x})|\mathrm{y}|^{\mathrm{p}-1} \quad(1<\mathrm{p}<\infty)
$$

is almost $\mathrm{z} \in \Omega$ and every $\mathrm{w}_{\mathrm{n}} \in \partial_{\mathrm{n}} \mathrm{j}\left(\mathrm{z}, \mathrm{y}_{1}, \ldots, \mathrm{y}_{\mathrm{i}}\right)$ and every $\mathrm{y}=\left(\mathrm{y}_{1}, \ldots, \mathrm{y}_{\mathrm{i}}\right) \in$ $\mathbb{R}^{N} \times \ldots \times \mathbb{R}^{N}$. Let us assume that $A=\left(A_{1}, \ldots, A_{i}\right): X_{1} \times \ldots \times X_{n} \rightarrow$ $\mathrm{L}^{\mathrm{p}}\left(\Omega, \mathbb{R}^{\mathrm{N}}\right) \times \ldots \times \mathrm{L}^{\mathrm{p}}\left(\Omega, \mathbb{R}^{\mathrm{N}}\right)$ and that $\mathrm{J} \circ \mathrm{A}: \mathrm{K}_{1} \times \ldots \times \mathrm{K}_{\mathrm{i}} \rightarrow \mathbb{R}$ is define by

$$
J(A u)=\int_{\Omega} j\left(z, A_{1} u_{1}(z), \ldots, A_{i} u_{i}(z)\right) d z .
$$

One can apply the Aubin-Clarke theorem [5] to get that

$$
\left.\mathrm{J}_{\mathrm{n}}{ }^{0}\left(\mathrm{Au} ; \mathrm{A}_{\mathrm{n}} \mathrm{v}_{\mathrm{n}}\right)\right) \leq \int_{\Omega} \mathrm{J}_{\mathrm{n}}{ }^{0}\left(\mathrm{z}, \mathrm{A}_{1} \mathrm{u}_{1}(\mathrm{z}), \ldots, \mathrm{A}_{\mathrm{i}} \mathrm{u}_{\mathrm{i}}(\mathrm{z}) ; \mathrm{A}_{\mathrm{n}} \mathrm{v}_{\mathrm{n}}(\mathrm{z})\right) \mathrm{dz} .
$$

For every $n=\overline{1,1}$ and $v_{n} \in X_{n}$. Since $H_{n}: X_{1} \times \ldots \times X_{i} \in \mathbb{R} \cup\{+\infty\}$ is a non negative and continuous on $K_{n}$. Then

$$
\begin{aligned}
& \mathrm{H}_{\mathrm{n}}\left(\mathrm{u}_{1}, \ldots, \mathrm{u}_{\mathrm{i}}\right) \mathrm{J}_{\mathrm{n}}{ }^{0}\left(\mathrm{Au} ; \mathrm{A}_{\mathrm{n}} \mathrm{v}_{\mathrm{n}}\right) \\
& \leq \mathrm{H}_{\mathrm{n}}\left(\mathrm{u}_{1}, \ldots, \mathrm{u}_{\mathrm{i}}\right) \int_{\Omega} \mathrm{J}_{\mathrm{n}}{ }^{0}\left(\mathrm{z}, \mathrm{A}_{1} \mathrm{u}_{1}(\mathrm{z}), \ldots, \mathrm{A}_{\mathrm{i}} \mathrm{u}_{\mathrm{i}}(\mathrm{z}) ; \mathrm{A}_{\mathrm{n}} \mathrm{v}_{\mathrm{n}}(\mathrm{z})\right) \mathrm{dz} .
\end{aligned}
$$

Corollary 4.2. Assume that the nonempty, bounded, closed and convex set $K_{n} \subset X_{n}$ for every $n=\overline{1,1}$. If the conditions $H_{F}, H_{T}, H_{i}, H_{H}$ and $\eta\left(u_{n}, v_{n}\right)=$ $v_{n}-u_{n}$ are satisfies. Then the following system of nonlinear quasihemivariational inequalities admits at least one solution. Finding $\left(\mathrm{u}_{1}, \ldots, \mathrm{u}_{\mathrm{i}}\right) \in$ $\mathrm{K}_{1} \times \ldots \times \mathrm{K}_{\mathrm{i}}$, in which 


\section{University of Thi-Qar Journal Vol.11 No.4 DEC 2016}

Web Site: https://jutq.utq.edu.iq/index.php/main Email: journal@jutq.utq.edu.iq

$$
\begin{aligned}
& \left\langle\mathrm{T}_{1}\left(\mathrm{u}_{1}, \ldots, \mathrm{u}_{\mathrm{i}}\right), \mathrm{v}_{1}-\mathrm{u}_{1}\right\rangle+\mathrm{H}_{1}\left(\mathrm{u}_{\mathrm{n}}\right) \int_{\Omega} \mathrm{j}_{1}^{0}\left(\mathrm{~A}_{1} \mathrm{u}_{1}(\mathrm{x}), \ldots, \mathrm{A}_{\mathrm{i}} \mathrm{u}_{\mathrm{i}}(\mathrm{x}) ; \mathrm{A}_{\mathrm{n}} \mathrm{v}_{\mathrm{n}}(\mathrm{x})\right. \\
& \left.-A_{n} u_{n}(x)\right) d x \geq\left\langle F_{1}\left(u_{1}, \ldots, u_{i}\right), v_{1}-u_{1}\right\rangle_{x_{1}} \\
& \begin{array}{llllllllllllllllll}
\ldots & \ldots & \ldots & \ldots & \ldots & \ldots & \ldots & \ldots & \ldots & \ldots & \ldots & \ldots & \ldots & \ldots & \ldots & \ldots & \ldots & (4.4)
\end{array} \\
& \left\langle\mathrm{T}_{\mathrm{i}}\left(\mathrm{u}_{1}, \ldots, \mathrm{u}_{\mathrm{i}}\right), \mathrm{v}_{\mathrm{i}}-\mathrm{u}_{\mathrm{i}}\right\rangle+\mathrm{H}_{\mathrm{i}}\left(\mathrm{u}_{\mathrm{n}}\right) \int_{\Omega}^{\mathrm{j}_{\mathrm{i}}}{ }^{0}\left(\mathrm{~A}_{1} \mathrm{u}_{1}(\mathrm{x}), \ldots, \mathrm{A}_{\mathrm{i}} \mathrm{u}_{\mathrm{i}}(\mathrm{x}) ; \mathrm{A}_{\mathrm{n}} \mathrm{v}_{\mathrm{n}}(\mathrm{x})\right. \\
& \left.-A_{n} u_{n}(x)\right) d x \geq\left\langle F_{i}\left(u_{1}, \ldots, u_{i}\right), v_{i}-u_{i}\right\rangle_{X_{i}} .
\end{aligned}
$$

For all $\left(v_{1}, \ldots, v_{i}\right) \in K_{1} \times \ldots \times K_{i}$.

It is worth mentioning that this kind of inequalities was studied by several authors (see $[10,22])$. 


\section{References}

[1] M. Alimohammady and A. E. Hashoosh, Existence theorems for $\alpha(u, v)$-monotone of nonstandard hemivariational inequality, Advances in Math. 10(2) (20(15) 3205-3212.

[2] B. Alleche and V. Radulescu, Equilibrium problem techniques in the qualitative analysis of quasi-hemivariational inequalities. Accepted. To appear in Optimization (2014).

[3] B. Alleche,V. Radulescu, M. Sebaoui, The Tikhonov regularization for equilibrium problems and applications to quasi-hemivariational inequalities, Optim., 9 (2015) 483-503.

[4] I. Andrei and N. Costea,Nonlinear hemivariational inequalities and applications to non- smooth mechanics,Adv. Nonlinear Var. Inequal. 13 (2010) 1-17.

[5] J. P. Aubin, and F. H. Clarke,Shadow prices and duality for a class of optimal control problems,SIAM J. Control Optim. 17 (1979) 567-586.

[6] M. Berger,Nonlinearity and Functional Analysis Academic Press,New York (1977).

[7] S. Carl,V. Khoi Le, and D. Motreanu,Nonsmooth Variational Problems and Their Inequal- ities,Springer Monographs in Mathematics,Springer,New York, (2007).

[8] F. H. Clarke, Optimization and Nonsmooth Analysis, Wiley (1983).

[9] C. Costea and V. Radulescu,Inequality problems of quasihemivariational type involving set-valued operators and a nonlinear term J. Glob. Optim. 52 (2012) 743-756.

[10] N. Costea, C. Varga,Systems of nonlinear hemivariational inequalities and applications, Topological Methods in Nonlinear Analysis. 1 (2003) 39-67. 
[11] K. Fan, Some properties of convex sets related to fixed point theorems. Math. Ann. 266 (1984) 519-537.

[12] A. E. Hashoosh, M. Alimohammady and M. K. Kalleji, Existence Results for Some Equilibrium Problems involving a-Monotone Bifunction, International Journal of Mathematics and Mathematical Sciences, 2016 (2016) 1-5.

[13] A. E. Hashoosh and M. Alimohammady, On well-posedness of generalized equilibrium problems involving $\alpha$-monotone bifunction, Journal of Hyperstructures, accepted.

[14] A. E. Hashoosh and M. Alimohammady, Existence and uniqueness results for a nonstandard variationl-hemivariational inequalities with application, Int. J. Industrial Mathematics (2016), accepted.

[15] S. Mig,rski,A. Ochal and M. Sofonea, Solvability of dynamic antiplane frictional contact problems for viscoelastic cylinders, Nonlinear Anal. 10 (2009) 3738-3748.

[16] S. Migrrski, A. Ochal and M. Sofonea, Weak solvability of antiplane frictional contact problems for elastic cylinders, Nonlinear Anal. 1 (2010) 172-183.

[17] J. Nash, Non-cooperative games, Annals of Mathemat-ics, 54 (1951) 268-295.

[18] P. Panagiotopoulos, Hemivariational Inequalities: Applications to Mechanics and Engineering, Springer-Verlag, New York Boston/Berlin, (1993).

[19] P. D. Panagiotopoulos, Nonconvex energy functions. Hemivariational inequalities and sub-stationarity principles, Acta Mech. 42 (1983) 160-183.

[20] N. S. Papageorgiou and S. T. Kyritsi-Yiallourou, Handbook of Applied Analysis. Advances in Mechanics and Mathematics, Springer, Dordrecht 19 (2009). 
[21] V. Radulescu, D. Repovs; Partial Differential Equations with Variable Exponents: Varia- tional Methods and Qualitative Analysis, CRC Press, Taylor Francis Group,Boca Raton FL (2015).

[22] D. Repovs and C.Varga, A Nash type solution for hemivariational inequality systems, Non- linear Analysis 74 (2011) 5585-5590.

[23] R. T. Rockefeller, Convex Analysis, Princeton University Press, New Jersey (1970).

[24] R. F. Susan-Resigaa, S. Munteanb, A. Stuparua, A. I. Bosioca, C. Tanasa and C. Ighiana, A variational model for swirling flow states with stagnant region, European Journal of Mechanics, 55 (2016) 104115.

[25] E. Tarafdar, A fixed point theorem equivalent to the Fan Knaster Kuratowski Mazurkiewicz Theorem, J. Math. Anal. Appl. 2 (1987) 475-479.

[26] R. U. Verma, A-monotonicity and its role in nonlinear variational inclusions, J. Optim. Theory Appl. 129 (2006) 457-467. 